\title{
A novel therapeutic strategy of personalized medicine based on anti-epidermal growth factor receptor monoclonal antibodies in patients with metastatic colorectal cancer
}

\author{
NOBUHISA MATSUHASHI, TAKAO TAKAHASHI, SATOSHI MATSUI, TOSHIYUKI TANAHASHI, \\ HISASHI IMAI, YOSHIHIRO TANAKA, KAZUYA YAMAGUCHI and KAZUHIRO YOSHIDA \\ Department of Surgical Oncology, Gifu University School of Medicine, Gifu 501-1194, Japan
}

Received October 24, 2017; Accepted January 24, 2018

DOI: $10.3892 /$ ijo.2018.4322

\begin{abstract}
Achieving tumor shrinkage may be a clinically relevant improvement in the treatment of metastatic colorectal cancer (mCRC). The present study attempted to evaluate early tumor shrinkage (ETS) and deepness of response over 6-8 courses of therapy, which were assessed previously in first-line trials of anti-epidermal growth factor receptor (EGFR) monoclonal antibodies. A total of 37 patients with mCRC that was considered unresectable or borderline resectable were enrolled in the study. Patients exhibited the wild-type RAS gene, and anti-EGFR monoclonal antibodies were used as the first-line treatment in the Department of Surgical Oncology at Gifu University School of Medicine (Gifu, Japan) between January 2010 and March 2017. Tumor shrinkage and other characteristics were evaluated according to the Response Evaluation Criteria In Solid Tumors (RECIST) classifications (version 1.1). The 3-year overall survival (OS) rate was $>60.0 \%$ for all cases $(n=37)$. The mean tumor shrinkage rate in the right side of the colon according to the RECIST classifications was $-11.1 \%$, whereas that for CRC on the left side showed a statistically significant difference at $-54.0 \%(\mathrm{P}=0.042)$. In addition, the rates of OS for stable disease + progressive disease compared with partial response + complete response, and those of OS for conversion therapy compared with non-conversion therapy were significantly different (both $\mathrm{P}<0.001)$. Carcinoembryonic antigen $(\mathrm{CEA})$ was suggested to be a possible predictive factor for convalescence due to the $50 \%$ drop in its value after the 6-8 courses of therapy. Overall,
\end{abstract}

Correspondence to: Dr Kazuhiro Yoshida, Department of Surgical Oncology, Gifu University School of Medicine, 1-1 Yanagido, Gifu 501-1194, Japan

E-mail:kyoshida@gifu-u.ac.jp

Key words: anti-epidermal growth factor receptor monoclonal antibodies, metastatic colorectal cancer, early tumor shrinkage, carcinoembryonic antigen, carbohydrate antigen 19-9 the predictive performance of ETS with respect to PFS and OS is at least as good as the standard RECIST response, with the advantage of an earlier assessment, and this may improve convalescence, with CEA as a marker in support of ETS over a clinical treatment course. In RAS wild-type patients, it is important to evaluate the rate of tumor shrinkage from the beginning of the first-line treatment until 6-8 courses of antiEGFR monoclonal antibodies have been administered.

\section{Introduction}

Colorectal cancer (CRC), the third most common malignant tumor in humans, is a major cause of cancer-associated mortality in the West. Metastatic tumors are present in 40-50\% of patients newly diagnosed with metastatic CRC (mCRC) (1), and their prognosis remains poor. The standard first-line chemotherapy for mCRC comprises a combination of fluorouracil (5-FU) and folinic acid with either oxaliplatin (FOLFOX-4 and FOLFOX-6 regimens) or irinotecan (FOLFIRI and AIO regimens) (2). The first-line treatment of the anti-vascular endothelial growth factor (VEGF) monoclonal antibody bevacizumab for $\mathrm{mCRC}$ has also been widely used with good effect, as shown in the pivotal AVF2107g and NO16966 trials (3-5). In addition, the combinations of irinotecan- or oxaliplatin-based chemotherapy with epidermal growth factor receptor (EGFR) inhibitors (cetuximab and panitumumab) are other standard first-line treatments for mCRC. A similar efficacy of FOLFIRI and FOLFOX has been shown in patients treated in this manner (6-9). In particular, these drugs are being investigated in terms of early tumor shrinkage (ETS) and deepness of response in unresectable-type and borderline resectable-type cancer with liver metastasis treated with anti-EGFR monoclonal antibodies (10), as the CALGB/SWOG 80405 trial showed no superiority in overall survival (OS) between anti-VEGF monoclonal antibodies and anti-EGFR monoclonal antibodies (11). Currently, the superiority of treatment with anti-EGFR monoclonal antibodies over that of anti-VEGF monoclonal antibodies remains confusing. The present study evaluated the possibility of proposing a novel treatment strategy for personalized medicine in which anti-EGFR monoclonal antibodies are used positively as a first-line treatment. 


\section{Patients and methods}

The present study included 37 patients with mCRC that was considered unresectable or borderline resectable who were confirmed to have the wild-type RAS gene. The patients underwent administration of first-line treatment with antiEGFR monoclonal antibodies in the Department of Surgical Oncology of Gifu University School of Medicine (Gifu, Japan) between January 2010 and March 2017. Written informed consent was obtained from all patients enrolled in the present study. The study protocol conformed to the ethical guidelines of the 1975 Declaration of Helsinki and the guidelines of the regional Ethics Committees of Zurich and Basel, Switzerland, following approval by the Institutional Review Board of the Gifu University Graduate School of Medicine.

The demographics and disease characteristics of the patients were recorded (Table I). Tumor shrinkage was evaluated according to the Response Evaluation Criteria In Solid Tumors (RECIST) version 1.1 classifications for these 37 patients who underwent first-line treatment consisting of 6-8 courses of FOLFOX plus anti-EGFR monoclonal antibody therapy.

RECIST guideline (version 1.1). Currently, computed tomography (CT) is the best and most reproducible method to measure lesions when assessing response. The RECIST guideline defines measurability of lesions on CT scan based on a CT slice thickness of $5 \mathrm{~mm}$ or less. When the CT slice thickness is $>5 \mathrm{~mm}$, the minimum size of a measurable lesion must be at least twice the slice thickness.

Evaluation of target lesions. The following definitions were applied for the evaluation of the target lesions. Complete response (CR): The disappearance of all target lesions. Any pathological lymph nodes (whether target or non-target) must have a reduction in the short axis to $<10 \mathrm{~mm}$. Partial response (PR): At least a 30\% decrease in the sum of the diameters of the target lesions as compared with the baseline sum diameters. Progressive disease (PD): At least a 20\% increase in the sum of the diameters of the target lesions as compared with the smallest sum in the study (this includes the baseline sum if that is the smallest in the study). In addition to the relative increase of $20 \%$, the sum must also show an absolute increase of at least $5 \mathrm{~mm}$. The appearance of one or more new lesions is also considered progression. Stable disease (SD): Neither sufficient shrinkage to qualify for PR nor sufficient increase to qualify for PD as compared with the smallest sum diameters in the study.

The present study also investigated the association between the predictive value of pre-chemotherapy changes in the levels of carcinoembryonic antigen (CEA) and carbohydrate antigen 19-9 (CA19-9), by determining whether there was a 50\% drop in their values after the 6-8 courses of therapy, and the clinical outcome following the anti-EGFR monoclonal antibody-based treatment regimen.

Statistical analysis. All data are presented as the mean \pm standard deviation. Student's t-test, Wilcoxon's signed-rank test, the Kaplan-Meier method, the log-rank test and Pearson's product-moment correlation coefficient were used to evaluate the data to determine statistical significances. A two-sided
P-value of $<0.05$ was considered to indicate a statistically significant difference. All statistical analyses were performed with SPSS 11.5J software (SPSS Japan, Inc., Tokyo, Japan).

\section{Results}

The study consisted of 37 patients with mCRC (27 men and 10 women; mean age, 62.1 \pm 9.4 years). Primary tumor locations in the 37 patients were as follows: The cecum in $2(5.4 \%)$ patients, the ascending colon in $5(13.5 \%)$ patients, the transverse colon in $4(10.8 \%)$ patients, the descending colon in $2(5.4 \%)$ patients, the sigmoid colon in 11 (29.7\%) patients, the rectosigmoid rectum in $5(13.5 \%)$ patients and the rectum above-below the peritoneal reflection in $8(21.6 \%)$ patients. Pathological types in the 37 patients were categorized as follows: 1 (2.7\%) patient with papillary adenocarcinoma, $6(16.2 \%)$ patients with well-differentiated adenocarcinoma, 27 (73.0\%) patients with moderately differentiated adenocarcinoma, 2 (5.4\%) patients with poorly differentiated adenocarcinoma and 1 (2.7\%) patient with mucinous adenocarcinoma. Metastatic sites of the 37 patients included the liver in 29 (78.4\%) patients, the lungs in $7(18.9 \%)$ patients, the spleen in $1(2.7 \%)$ patient, the lymph nodes in $9(24.3 \%)$ patients and the bones in $2(5.4 \%)$ patients. The number of metastatic sites included 1 site in 24 (64.9\%) patients, 2 sites in 8 (21.6\%) patients, 3 sites in 4 (10.8\%) patients and 4 sites in $1(2.7 \%)$ patient (Table I).

Curves showing cumulative survival are presented in Fig. 1. The 3-year OS rate was $>60.0 \%$ in all patients $(n=37)$. Patients receiving the anti-EGFR monoclonal antibodies as first-line treatment did not reach the median survival time. These 3 -year OS data are meaningful as the OS time in a recent study reached only slightly more than 30 months (11).

The OS rates for right-sided CRC compared with left-sided CRC were not significantly different $(\mathrm{P}=0.235)$. However, the OS rates for left-sided CRC treated with the anti-EGFR monoclonal antibodies as first-line treatment tended to be better than those for right-sided CRC (Fig. 2). The mean tumor shrinkage rate in right-sided CRC according to the classification of the RECIST guidelines (version 1.1) was $-11.1 \%$, whereas that for the left-sided CRC was significantly different at $-54.0 \%(\mathrm{P}=0.042)$ (Fig. 3). In addition, the OS rates for $\mathrm{SD}+\mathrm{PD}$ compared with $\mathrm{PR}+\mathrm{CR}$ were significantly different $(\mathrm{P}<0.001)$ (Fig. 4).

The overall median tumor shrinkage rate of all patients according to the RECIST guideline (version 1.1) classification was $-49.6 \%$. The estimated median shrinkage rate in the 29 patients with CR+PR was $-60.3 \%$. In addition, the 8 patients with SD+PD did not show apparent shrinkage, with an estimated median shrinkage rate of $-0.3 \%$. Waterfall plots dividing $\mathrm{PR}+\mathrm{CR}$ and $\mathrm{SD}+\mathrm{PD}$ and left-sided and rightsided CRC location are shown in Fig. 5. Characteristics of patient who received first-line treatment with FOLFOX plus anti-EGFR monoclonal antibodies are presented by RECIST (version 1.1) response to chemotherapy according to changes in CEA and CA19-9 in Table II.

The OS rates for patients treated with conversion therapy, which was defined as surgical therapy with R0 or R1 margins in unresectable and marginally resectable metastases, compared with patients treated with non-conversion therapy were significantly different $(\mathrm{P}<0.001)($ Fig. 6). In addition, CEA 


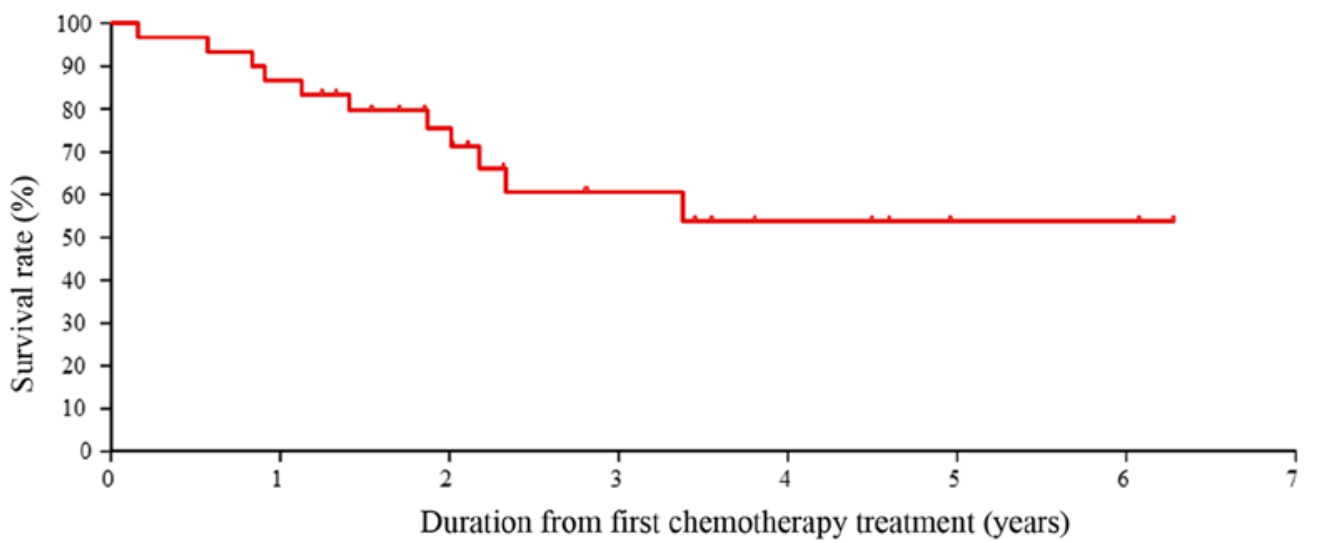

Figure 1. Cumulative survival curves of all metastatic colorectal cancer cases ( $\mathrm{n}=37)$. Overall survival of patients was calculated.

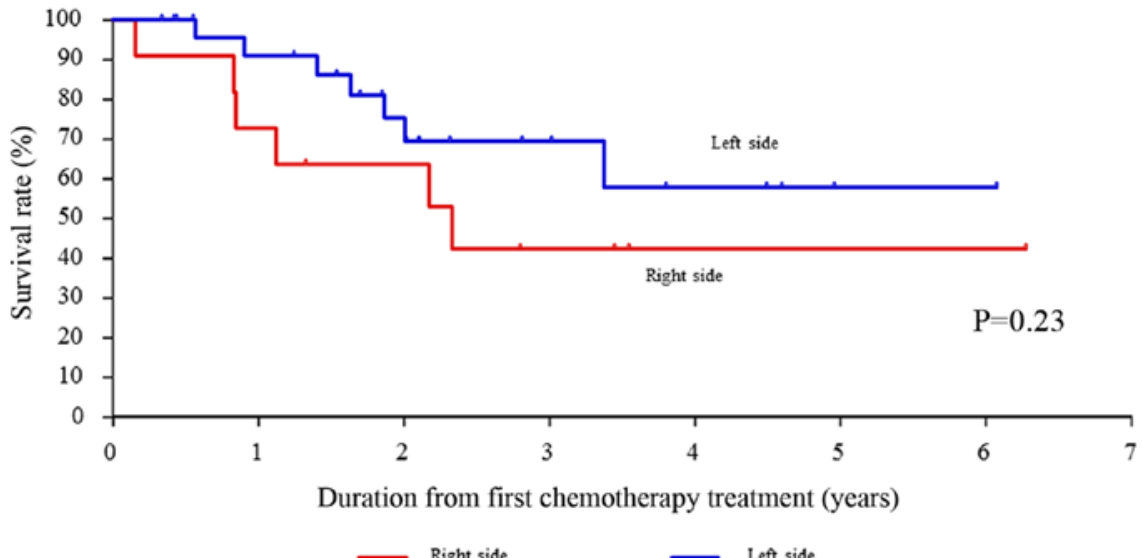

Figure 2. Cumulative survival curves showing the rates of overall survival between the patients with right-sided colon and those with left-sided colorectal cancer.

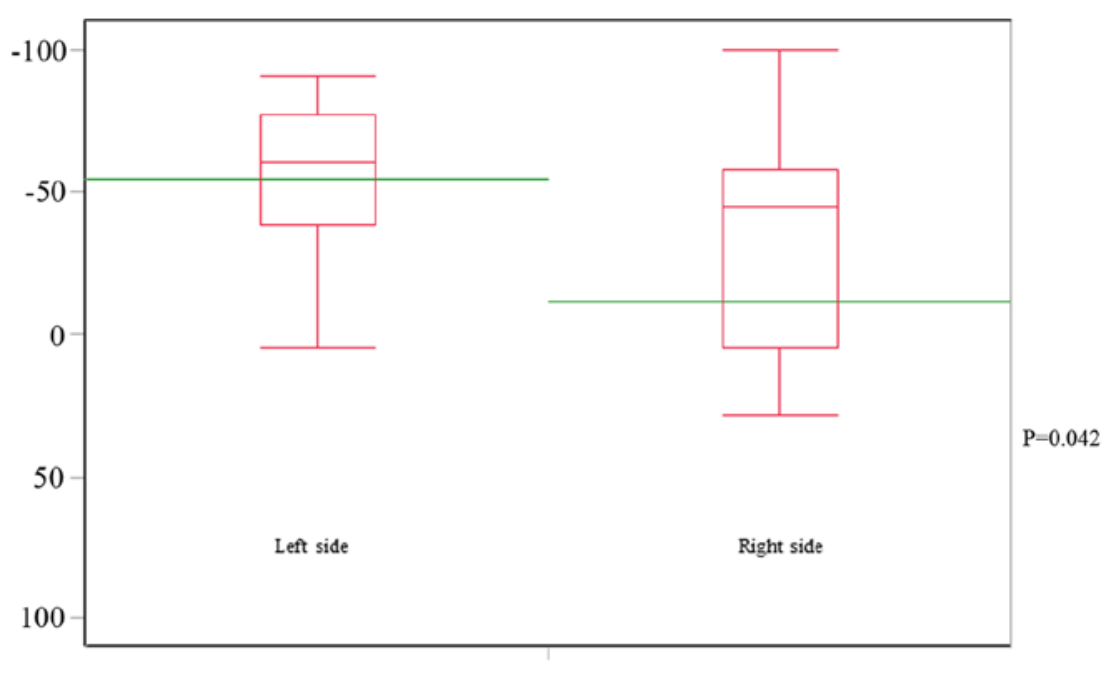

$54.0 \%$ reduced

$11.1 \%$ reduced

Figure 3. Mean tumor shrinkage rate in right-sided and left-sided colorectal cancer according to the classification of the Response Evaluation Criteria In Solid Tumors guidelines.

was suggested as a possible predictive factor for survival due to the significant $50 \%$ drop in its value $(\mathrm{P}<0.05)$ following the 6-8 courses of therapy (Fig. 7). However, the level of CA19-9 did not decrease by $50 \%$ following the $6-8$ courses of therapy, suggesting that it was not likely to be a predictive factor of survival (Fig. 8). 
Table I. Characteristics of 37 patients with metastatic colorectal cancer who received anti-epidermal growth factor receptor monoclonal antibodies as first-line treatment.

\begin{tabular}{|c|c|}
\hline Characteristics & Value \\
\hline \multicolumn{2}{|l|}{$\operatorname{Sex}, \mathrm{n}(\%)$} \\
\hline Female & $10(27.0)$ \\
\hline Male & $27(73.0)$ \\
\hline \multicolumn{2}{|l|}{ Age, years } \\
\hline Mean \pm SD (range) & $62.1 \pm 9.4(39-77)$ \\
\hline \multicolumn{2}{|l|}{ Primary tumor, n (\%) } \\
\hline $\mathrm{C}$ & $2(5.4)$ \\
\hline A & $5(13.5)$ \\
\hline $\mathrm{T}$ & $4(10.8)$ \\
\hline $\mathrm{D}$ & $2(5.4)$ \\
\hline $\mathrm{S}$ & $11(29.7)$ \\
\hline Rs & $5(13.5)$ \\
\hline $\mathrm{Ra}-\mathrm{b}$ & $8(21.6)$ \\
\hline \multicolumn{2}{|l|}{ Pathology, n (\%) } \\
\hline pap & $1(2.7)$ \\
\hline tub1 & $6(16.2)$ \\
\hline tub2 & $27(73.0)$ \\
\hline por & $2(5.4)$ \\
\hline muc & $1(2.7)$ \\
\hline \multicolumn{2}{|l|}{ Metastatic site, n (\%) } \\
\hline Liver & $29(78.4)$ \\
\hline Lung & $7(18.9)$ \\
\hline Spleen & $1(2.7)$ \\
\hline Lymph node & $9(24.3)$ \\
\hline Peritoneal & $6(16.2)$ \\
\hline Bone & $2(5.4)$ \\
\hline \multicolumn{2}{|l|}{ Metastases, n (\%) } \\
\hline 1 & $24(64.9)$ \\
\hline 2 & $8(21.6)$ \\
\hline 3 & $4(10.8)$ \\
\hline 4 & $1(2.7)$ \\
\hline
\end{tabular}

SD, standard deviation; pap, papillary adenocarcinoma; tub1, well-differentiated adenocarcinoma; tub2, moderately differentiated adenocarcinoma; por, poorly differentiated adenocarcinoma; muc, mucinous adenocarcinoma; $\mathrm{C}$, cecum; A, ascending colon; T, transverse colon; D, descending colon; S, sigmoid colon; Rs, rectosigmoid; Ra-b, rectum above-below the peritoneal reflection.

\section{Discussion}

The combinations of irinotecan- or oxaliplatin-based chemotherapy with EGFR inhibitors (cetuximab and panitumumab) are standard first-line treatments for mCRC. A similar efficacy of FOLFIRI and FOLFOX has been shown in these patients (6-9).

The randomized phase III FIRE-3 trial, first reported by Heinemann et al (12) in 2014, aimed to compare the efficacy of FOLFIRI plus cetuximab with FOLFIRI plus bevacizumab
Table II. RECIST response to chemotherapy according to changes in CEA and CA19-9.

\begin{tabular}{lc}
\hline CEA change, $\%$ & \\
$<50$ & $15(40.5)$ \\
$\geq 50$ & $22(59.5)$ \\
CA $19-9$ change, $\%$ & \\
$<50$ & $25(67.6)$ \\
$\geq 50$ & $12(32.4)$ \\
Disease status ${ }^{\text {a }}$ & \\
CR & $1(2.7)$ \\
PR & $28(75.7)$ \\
SD & $6(16.2)$ \\
PD & $2(5.4)$ \\
Conversion therapy & \\
Yes & $19(51.4)$ \\
No & $18(48.6)$
\end{tabular}

aAccording to RECIST, version 1.1. CR, complete response; PR, partial response; PD, progressive disease; SD, stable disease; RECIST, Response Evaluation Criteria In Solid Tumors.

in 592 patients with KRAS-wild-type mCRC. A significant OS advantage was found in the patients treated with first-line FOLFIRI plus cetuximab, as their median OS time increased by almost 4 months [28.8 vs. 25.0 months; hazard ratio (HR), $0.77 ; \mathrm{P}=0.0164]$. Results from a sub-analysis that excluded patients with other activating mutations in the RAS family genes (all-RAS wild-type population) showed an increase in median OS time of 7.5-months: 33.1 months with FOLFIRI plus cetuximab vs. 25.6 months with FOLFIRI plus bevacizumab (HR, 0.70; P=0.011) $(13,14)$.

In the PEAK trial, 278 patients with previously untreated wild-type KRAS exon 2 (codons 12 and 13) tumors received either panitumumab plus modified 5-FU, leucovorin and oxaliplatin (mFOLFOX6) or bevacizumab plus mFOLFOX6. A pre-planned analysis of extended all-RAS genes (including exons 2, 3 and 4 of KRAS and NRAS) was also included. Despite the similar rates of progression-free survival (PFS) (HR, 0.87; $\mathrm{P}=0.353$ ), FOLFOX plus panitumumab showed a significant advantage in in terms of OS, with a 12-month survival gain in the all-RAS wild-type population (median OS, 41.3 vs. 28.9 months; $\mathrm{P}=0.058$ ). The present analysis is the first to evaluate the concepts of ETS and deepness of response, which were previously assessed in first-line trials that included anti-EGFR monoclonal antibodies (10)

Achieving tumor shrinkage may be clinically relevant when treating mCRC. Not only may it permit the secondary resection of metastatic lesions, which can provide a possible cure even in a subgroup of patients with metastasis, it may also improve or delay the occurrence of symptoms in patients with aggressive diseases and high tumor load (15). In the present study, the rates of OS for conversion therapy compared with those for non-conversion therapy were significantly different $(\mathrm{P}<0.001)$. This result suggests that if it is performed concomitantly with surgery, conversion therapy will prolong the OS rate. 


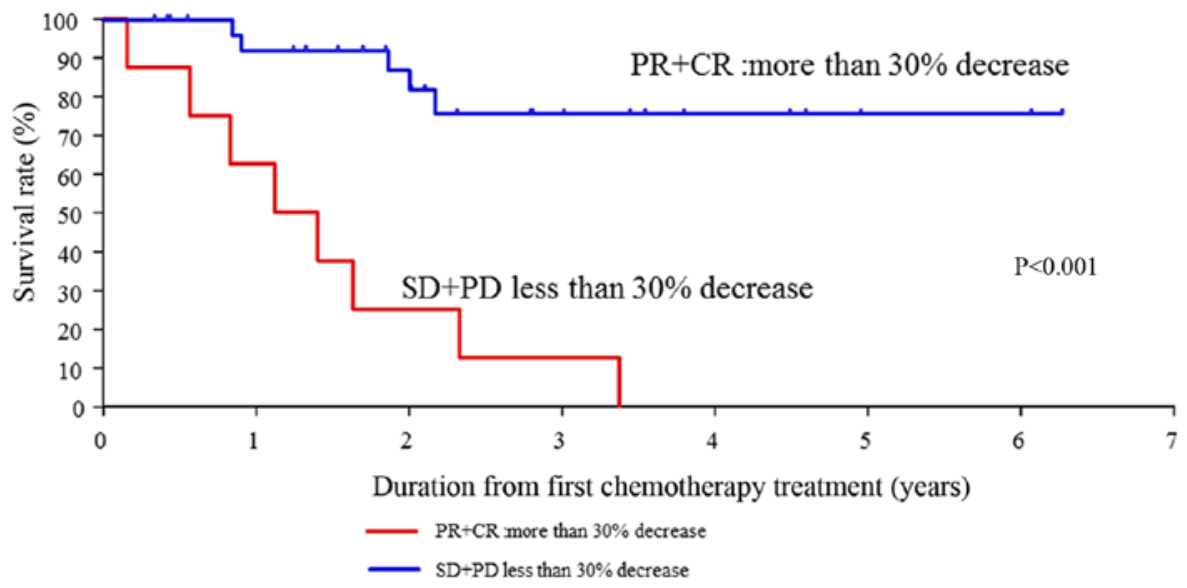

Figure 4. Cumulative survival curves showing the OS rates for patients with SD+PD compared with those with PR+CR. Differences were statistically significant $(\mathrm{P}<0.001)$. $\mathrm{CR}$, complete response; $\mathrm{PR}$, partial response; $\mathrm{PD}$, progressive disease; $\mathrm{SD}$, stable disease.
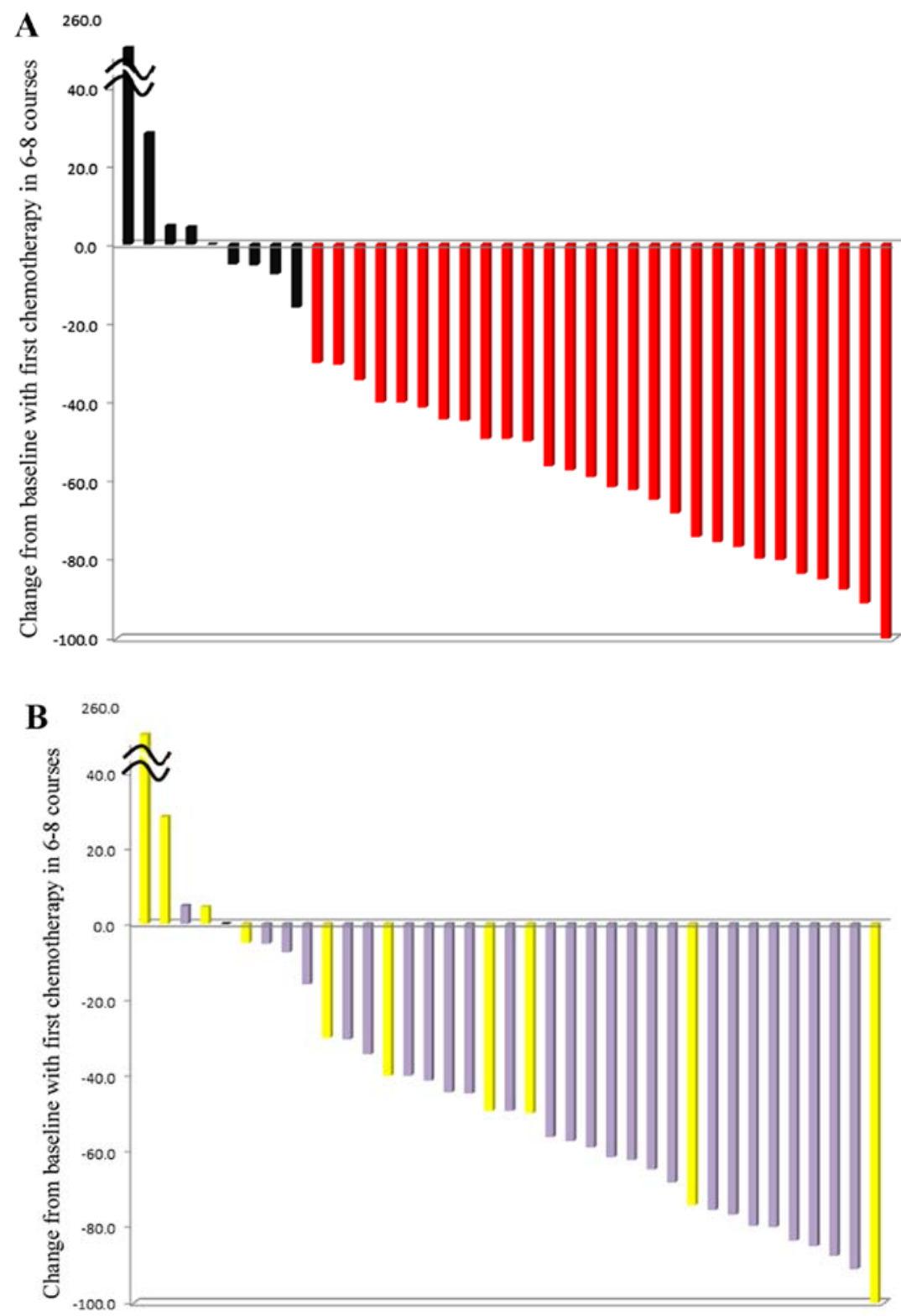

Figure 5. Cumulative survival curves. (A) The cumulative survival rate of the 29 patients with $\mathrm{CR}+\mathrm{PR}$ was estimated to be $-60.3 \%$, whereas that in the 8 patients with stable disease + progressive disease was estimated to be $-0.3 \%$, indicating no shrinkage. (B) The cumulative survival rates for right-sided CRC (yellow) and left-sided CRC (purple). The results indicated that anti-EGFR monoclonal antibodies can be expected to result in an effect in right-sided CRC. CRC, colorectal cancer; EGFR, epidermal growth factor receptor. 


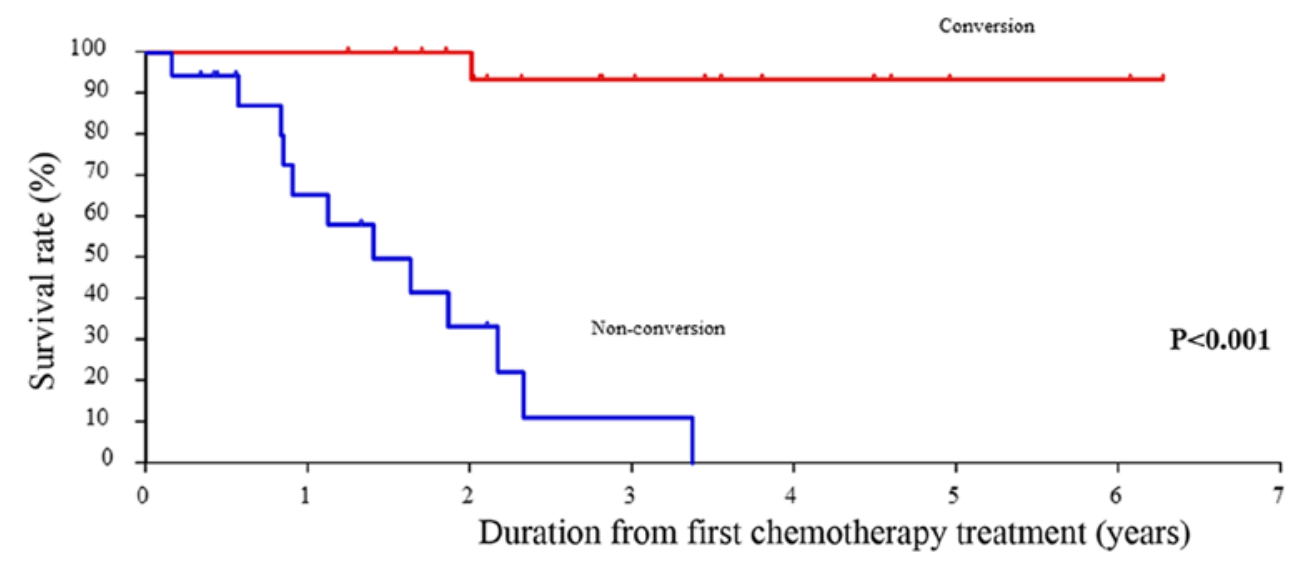

Conversion $\quad$ Non-conversion

Figure 6. Cumulative survival curves showing the overall survival rates for conversion therapy compared with non-conversion therapy. Differences were statistically significant $(\mathrm{P}<0.001)$.

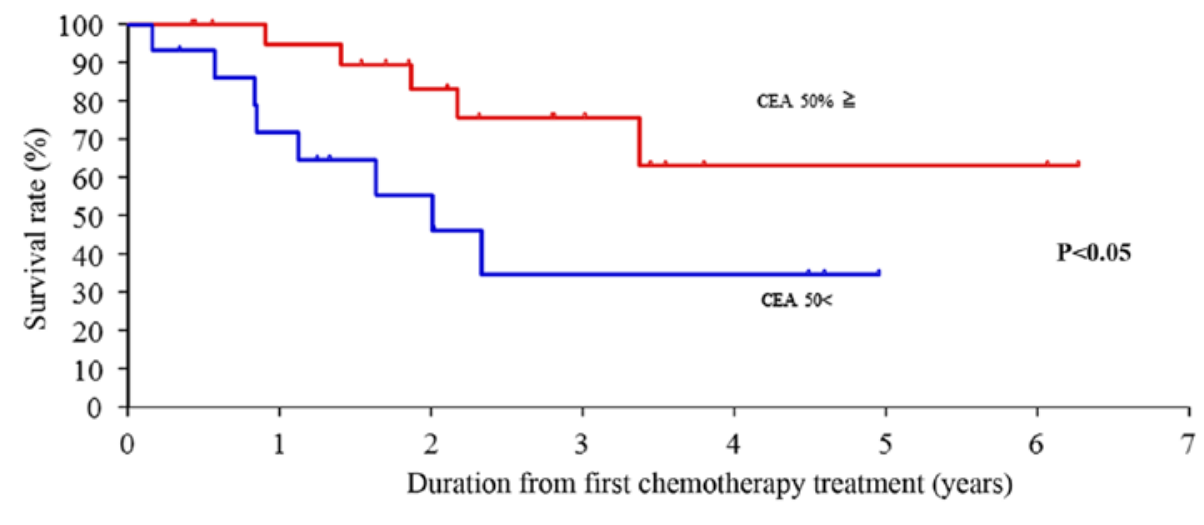

Figure 7. Cumulative survival curves showing that the 50\% drop in CEA level suggest it to be a possible predictive factor for survival following 6-8 courses of therapy. CEA, carcinoembryonic antigen.

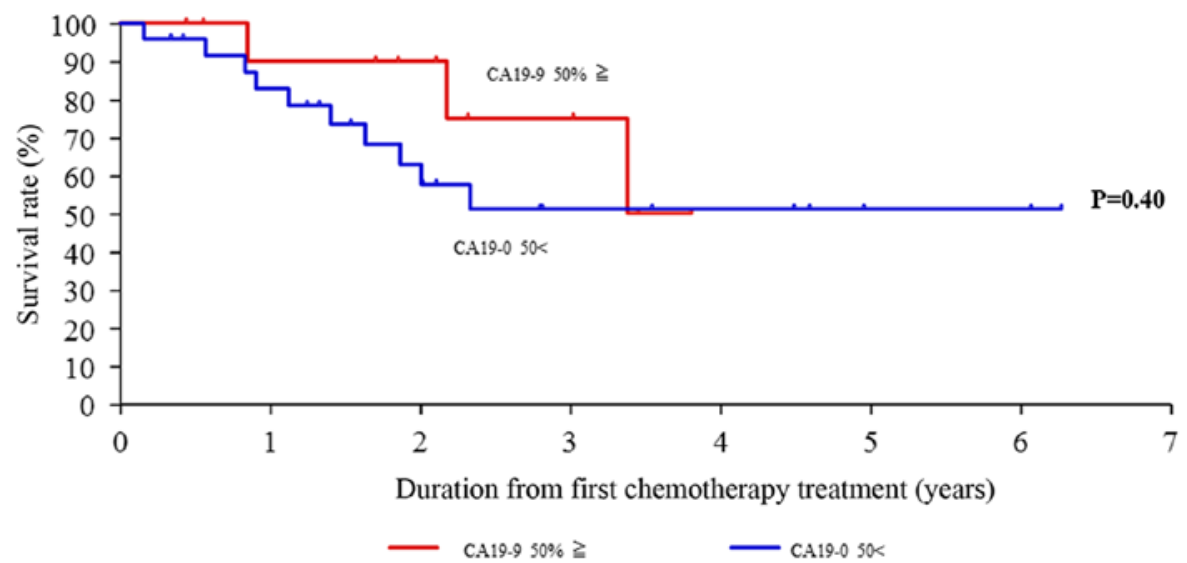

Figure 8. Cumulative survival curves showing the lack of a 50\% drop in CA19-9, suggesting that it is not likely to be a possible predictive factor for survivor following 6-8 courses of therapy. CA19-9, carbohydrate antigen 19-9.

On the basis of these results, $>1,100$ KRAS wild-type patients were enrolled in the CALGB 80405 trial, and among them, 526 all-RAS wild-type patients underwent a separate subanalysis to assess study endpoints. No advantage for cetuximab in terms of either OS or PFS could be confirmed. In particular, the median PFS time in the all-RAS wild-type population was $\sim 11$ months for the two arms (HR, 1.1; $\mathrm{P}=0.31$ ), and the median OS time was 31-32 months (HR, 0.9; $\mathrm{P}=0.40$ ) (16).

The predictive performance of ETS is at least as good as the standard RECIST response with respect to PFS, OS and 


\section{Conversion therapy possible (liver and lung)}

$\left\langle 1^{\text {st }}\right.$ line $>\quad\left\langle 2^{\text {nd }}\right.$ line $>$

6-8courses shrinkage rate $\geqq 30 \% \mathrm{PR}$

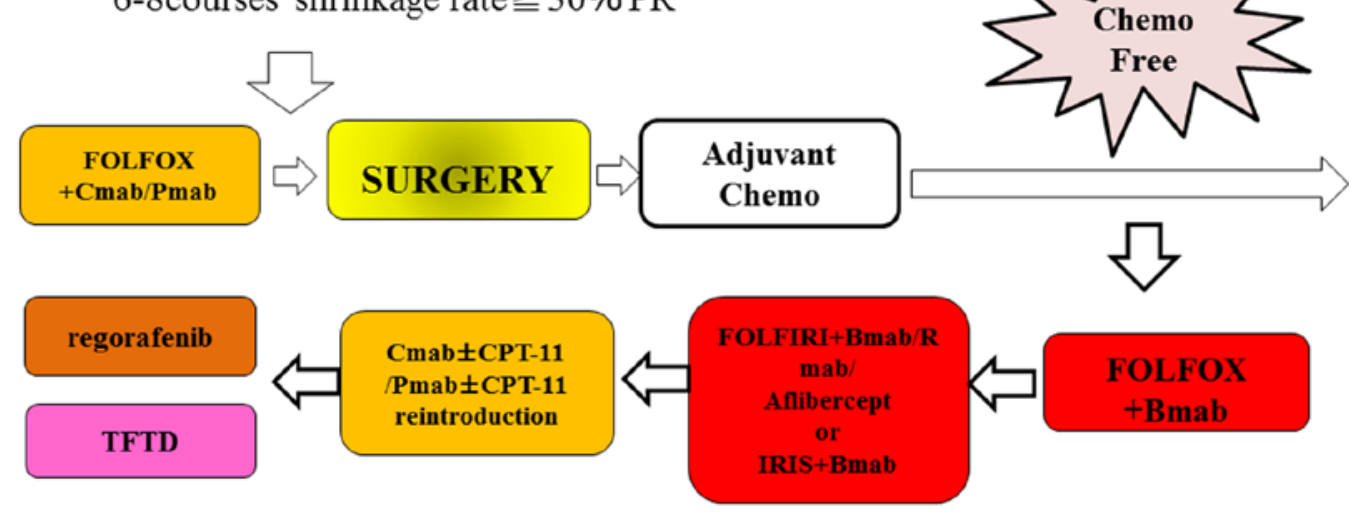

Figure 9. A novel treatment strategy in metastatic colorectal cancer cases indicating when conversion therapy (liver and lung) is possible. Cmab, cetuximab; Pmab, panitumumab; Bmab, bevacizumab; Rmab, ramucirumab; TFTD, TAS 102; PR, partial response; CPT-11, irinotecan.

\section{Conversion therapy possible (liver and lung)}

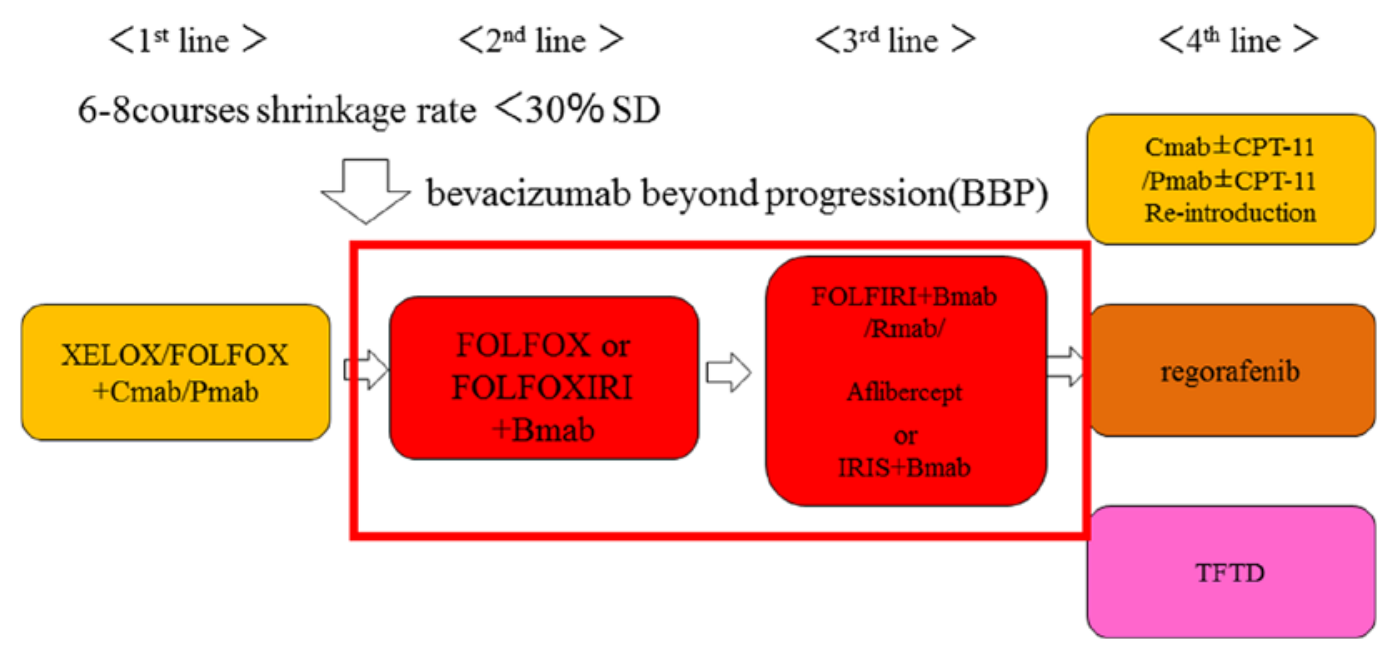

Figure 10. A novel strategy in metastatic colorectal cancer cases indicating when conversion therapy (liver and lung) is not possible. BBP, bevacizumab beyond progression; Cmab, cetuximab; Pmab, panitumumab; Bmab, bevacizumab; Rmab, ramucirumab; TFTD, TAS 102; SD, stable disease; CPT-11, irinotecan; IRIS, TS-1 + irinotecan.

post-progression survival, and it allows for earlier assessment. Therefore, it would be extremely appealing to use this endpoint in clinical trials to expedite drug development and to potentially guide therapeutic decisions. As the surrogacy of ETS has yet to be shown at trial level (17), the present study evaluated whether ETS could be a significant prognosticator of OS.

The mean tumor shrinkage rate of all patients according to the RECIST guideline (version 1.1) classification was $-49.6 \%$. The rate in the 29 patients with $\mathrm{CR}+\mathrm{PR}$ was estimated to be $-60.3 \%$, whereas that in the 8 patients with SD+PD was estimated to be $-0.3 \%$, indicating no shrinkage.

CEA and CA19-9 are used as surrogate markers of predictive factors in mCRC. Although CEA is a glycoprotein present in normal mucosal cells, its elevation is observed in a number of different malignancies (18-22). CA19-9 was first identified as a mucin-like product in a human CRC cell line (23). The importance of an elevated serum level of CA19-9 as a useful marker for diagnosing adenocarcinoma of the upper gastrointestinal tract and for monitoring colon tumors has been suggested by a number of studies (24-26). CEA and CA19-9 are considered to be useful as tumor markers in the assessment of prognosis, in the detection of recurrence and in the monitoring of the treatment of patients with CRC.

Prager et al (27) first reported that the canonical biomarker CEA, a monitor of adenocarcinoma growth and treatment efficacy, may also be a predictive marker of anti-VEGF-based combination therapies in addition to its biological role $(28,29)$. The described effects of CEA on treatment outcome were clearly limited to bevacizumab-based treatment. In addition, Ocvirk et al (30) evaluated CEA and treatment efficacy in a cetuximab-based cohort in comparison to a bevacizumab 
cohort. Negative results in the control cohort indicated that baseline CEA levels were predictive only for bevacizumabbased treatment, suggesting that for anti-VEGF monoclonal antibody treatment in $\mathrm{mCRC}$, CEA may be a specific marker of response and PFS. In the present study, CEA was suggested to be a predictor of convalescence, as its level dropped by $50 \%$ following the 6-8 courses of therapy. However, CA19-9 was not suggested to be a predictive factor, as its level did not decrease by $50 \%$ following the $6-8$ courses. These results were similar to those of numerous other studies (27-30).

It was suggested that patients with right-sided CRC had a worse rate of tumor reduction than patients with left-sided CRC. Recently, the location of the primary tumor, whether of right- or left-sided origin, has been investigated for its role in assisting in the prediction of outcomes. Median OS time with anti-EGFR monoclonal antibody treatment in CALGB/SWOG80405 trial (11) was significantly different between patients with CRC of right- and left-sided origins. Specifically, OS with anti-EGFR monoclonal antibody treatment was significantly worse for patients with CRC of right-sided origin. The present study also suggested that the shrinkage rate was worse in the patients with a right-sided versus left-sided origin (14). Anti-EGFR monoclonal antibodies have an effect on ETS and may improve OS in patients with mCRC. If anti-EGFR monoclonal antibodies are used for 6-8 courses, this may be useful as a predictor of convalescence. If SD is determined with treatment using anti-EGFR monoclonal antibodies, the switch to anti-VEGF monoclonal antibodies should be considered at the time of the 6- 8 course, as recommended by the RECIST guideline classification (version 1.1). Anti-EGFR monoclonal antibodies are not therefore not expected to be effective as first-line treatment, but their use as third-line treatment should be considered. This concept means the re-introduction of treatment using anti-EGFR monoclonal antibodies.

Identifying prognostic molecules that can predict the effectiveness of aggressive chemotherapy to prevent tumor relapse represents a challenge for clinical practice. The 2016 European Society for Medical Oncology (ESMO) guidelines (31) recommend that the best benefit-to-risk ratio can be obtained with a cytotoxic doublet plus an anti-EGFR antibody for patients with RAS wild-type disease; however, the combination of FOLFOXIRI with bevacizumab can also be considered (32). The TRIBE study reported that FOLFOXIRI plus bevacizumab, compared with FOLFIRI plus bevacizumab, resulted in significantly higher response rates (65 vs. 53\%) (32). On this basis, cytoreduction of the disease may prolong OS and PFS times. We recently reported that the overall rate of tumor shrinkage was highest during the first 50 days after the start of therapy and gradually decreased over the next 210 days subsequent to plateauing at 105 days $(33,34)$. The present results indicated that the effect of anti-EGFR monoclonal antibodies plus FOLFOX on tumor size is achieved at around 3 months (6-8 courses).

Taking into consideration the present results and the evidence in the literature, we believe that patients with RAS wild-type disease should still undergo 6-8 courses of therapy with anti-EGFR monoclonal antibodies plus FOLFOX. If tumor shrinkage shows a PR, these patients should continue on this same therapy. Also, it is possible to perform conversion therapy (surgery) in patients with unresectable or borderline resectable liver and lung $\mathrm{mCRC}$ (Fig. 9).

We thus propose a novel treatment strategy for personalized medicine in patients in whom anti-EGFR monoclonal antibodies are used as first-line treatment. If the rate of tumor shrinkage indicates PD, patients with RAS wild-type disease (BRAF mutant-type) may switch to the combination of FOLFOXIRI plus bevacizumab to provide a stronger therapeutic agent. If the rate of tumor shrinkage indicates SD, these patients may be switched to therapy with anti-VEGF monoclonal antibodies plus FOLFOX to provide a maintenance dose of the therapeutic agent (Fig. 10).

Recent National Comprehensive Cancer Network (35) and ESMO guidelines (31) have recommended that anti-PDL-1 antibody treatment should be used in patients with high microsatellite instability. Therefore, physicians should consider not only RAS-type disease, but also diseases with other individual genomic types.

In conclusion, at present in RAS wild-type patients, it is important to evaluate the rate of tumor shrinkage from the beginning of the first-line treatment until 6-8 courses of anti-EGFR monoclonal antibody administration have been completed, and it is important to determine whether conversion therapy is possible. In addition, it is important to determine whether anti-EGFR monoclonal antibodies are effective. In the case of localized liver metastasis, anti-VEGF monoclonal antibodies plus FOLFOXIRI may be used after 6-8 courses of anti-EGFR monoclonal antibodies plus FOLFOX in order to make the treatment more effective and obtain stable disease. In this case, we expect that the use of this novel strategy of anti-VEGF monoclonal antibodies plus FOLFOXIRI or bevacizumab beyond progression will be possible, and furthermore, that anti-VEGF monoclonal antibodies of the new generation, such as ramucirumab and aflibercept, will also be used. In addition, we expect that strategic treatments, including re-challenge and re-introduction with anti-EGFR monoclonal antibodies, will further prolong the OS and PFS times of these patients with mCRC.

\section{Acknowledgements}

Not applicable.

\section{Funding}

No funding was received.

\section{Availability of data and materials}

All data generated or analyzed during this study are included in this published article.

\section{Authors' contributions}

NM and TaT conceived the study and its design. NM, TaT, SM, ToT, HI, YT, KYa and KYo acquired the data. NM analyzed and interpreted the data and drafted the manuscript. NM, TaT, and KYo performed critical revision of the manuscript. KYo supervised the study. All authors have read and approved the final manuscript. 


\section{Ethics approval and consent to participate}

All procedures performed in studies involving human participants were in accordance with the ethical standards of the institutional and/or national research committee and with the 1975 Declaration of Helsinki and its later amendments or comparable ethical standards. Informed consent was obtained from all individual participants included in the study.

\section{Consent for publication}

Not applicable.

\section{Competing interests}

KYo has received honoraria for lectures from Chugai Pharmaceutical Co., Ltd., Taiho Pharmaceutical Co., Ltd., Takeda Pharmaceutical Co., Ltd., Eli Lilly and Company, Daiichi Sankyo Co.,Ltd., Ono Pharmaceutical Co., Ltd., Merck Serono Co., Ltd., Novartis Pharma K.K. and Sanofi K.K., and research funding from Ajinomoto Pharmaceutical Co., Ltd., Takeda Pharmaceutical Co., Ltd., Chugai Pharmaceutical Co., Ltd., Daiichi Sankyo Co., Ltd., Taiho Pharmaceutical Co., Ono Pharmaceutical Co., and Yakult Honsha Co., Ltd., outside the submitted work. TaT has received honoraria for lectures from Takeda Pharmaceutical Co., Ltd. All remaining authors declare that they have no conflicts of interest.

\section{References}

1. Fernandez FG, Drebin JA, Linehan DC, Dehdashti F, Siegel BA and Strasberg SM: Five-year survival after resection of hepatic metastases from colorectal cancer in patients screened by positron emission tomography with F-18 fluorodeoxyglucose (FDG-PET). Ann Surg 240: 438-450, 2004

2. O'Neil BH and Goldberg RM: Innovations in chemotherapy for metastatic colorectal cancer: An update of recent clinical trials. Oncologist 13: 1074-1083, 2008.

3. Grothey A, Hedrick EE, Mass RD, Sarkar S, Suzuki S, Ramanathan RK, Hurwitz HI, Goldberg RM and Sargent DJ Response-independent survival benefit in metastatic colorectal cancer: A comparative analysis of N9741 and AVF2107. J Clin Oncol 26: 183-189, 2008.

4. Tyagi P and Grothey A: Commentary on a phase III trial of bevacizumab plus XELOX or FOLFOX4 for first-line treatment of metastatic colorectal cancer: The NO16966 trial. Clin Colorectal Cancer 6: 261-264, 2006.

5. Arkenau HT, Arnold D, Cassidy J, Diaz-Rubio E, Douillard JY, Hochster H, Martoni A, Grothey A, Hinke A, Schmiegel W, et al: Efficacy of oxaliplatin plus capecitabine or infusional fluorouracil/leucovorin in patients with metastatic colorectal cancer: A pooled analysis of randomized trials. J Clin Oncol 26: 5910-5917, 2008.

6. Falcone A, Ricci S, Brunetti I, Pfanner E, Allegrini G, Barbara C, Crinò L, Benedetti G, Evangelista W, Fanchini L, et al; Gruppo Oncologico Nord Ovest: Phase III trial of infusional fluorouracil, leucovorin, oxaliplatin, and irinotecan (FOLFOXIRI) compared with infusional fluorouracil, leucovorin, and irinotecan (FOLFIRI) as first-line treatment for metastatic colorectal cancer: The Gruppo Oncologico Nord Ovest. J Clin Oncol 25: 1670-1676, 2007.

7. Van Cutsem E, Köhne CH, Hitre E, Zaluski J, Chang Chien CR, Makhson A, D'Haens G, Pintér T, Lim R, Bodoky G, et al: Cetuximab and chemotherapy as initial treatment for metastatic colorectal cancer. N Engl J Med 360: 1408-1417, 2009.

8. Bokemeyer C, Bondarenko I, Makhson A, Hartmann JT, Aparicio J, de Braud F, Donea S, Ludwig H, Schuch G, Stroh C, et al: Fluorouracil, leucovorin, and oxaliplatin with and without cetuximab in the first-line treatment of metastatic colorectal cancer. J Clin Oncol 27: 663-671, 2009.
9. Saltz LB, Clarke S, Díaz-Rubio E, Scheithauer W, Figer A, Wong R, Koski S, Lichinitser M, Yang TS, Rivera F, et al: Bevacizumab in combination with oxaliplatin-based chemotherapy as first-line therapy in metastatic colorectal cancer: A randomized phase III study. J Clin Oncol 26: 2013-2019, 2008.

10. Schwartzberg LS, Rivera F, Karthaus M, Fasola G, Canon JL, Hecht JR, Yu H, Oliner KS and Go WY: PEAK: A randomized, multicenter phase II study of panitumumab plus modified fluorouracil, leucovorin, and oxaliplatin (mFOLFOX6) or bevacizumab plus mFOLFOX6 in patients with previously untreated, unresectable, wild-type KRAS exon 2 metastatic colorectal cancer. J Clin Oncol 32: 2240-2247, 2014.

11. Venook AP, Niedzwiecki D, Lenz HJ, Innocenti F, Fruth B, Meyerhardt JA, Schrag D, Greene C, O'Neil BH, Atkins JN, et al: Effect of first-line chemotherapy combined with cetuximab or bevacizumab on overall survival in patients with KRAS wild-type advanced or metastatic colorectal cancer: A randomized clinical trial. JAMA 317: 2392-2401, 2017.

12. Heinemann V, von Weikersthal LF, Decker T, Kiani A, Vehling-Kaiser U, Al-Batran SE, Heintges T, Lerchenmüller C, Kahl C, Seipelt G, et al: FOLFIRI plus cetuximab versus FOLFIRI plus bevacizumab as first-line treatment for patients with metastatic colorectal cancer (FIRE-3): A randomised, openlabel, phase 3 trial. Lancet Oncol 15: 1065-1075, 2014

13. Stintzing S, Modest DP, Rossius L, Lerch MM, von WeikersthalLF, Decker T, Kiani A, Vehling-Kaiser U, Al-Batran SE, Heintges T, et al; FIRE-3 investigators: FOLFIRI plus cetuximab versus FOLFIRI plus bevacizumab for metastatic colorectal cancer (FIRE-3): A post-hoc analysis of tumour dynamics in the final RAS wild-type subgroup of this randomised open-label phase 3 trial. Lancet Oncol 17: 1426-1434, 2016.

14. Tejpar S, Stintzing S, Ciardiello F, Tabernero J, Van Cutsem E, Beier F, Esser R, Lenz HJ and Heinemann V: Prognostic and predictive relevance of primary tumor location in patients with RAS wild-type metastatic colorectal cancer: Retrospective analyses of the CRYSTAL and FIRE-3 trials. JAMA Oncol: Oct 10, 2016 (Epub ahead of print). doi: 10.1001/jamaoncol.2016.3797.

15. Folprecht G, Gruenberger T, Bechstein WO, Raab HR, Lordick F, Hartmann JT, Lang H, Frilling A, Stoehlmacher J, Weitz J, et al: Tumour response and secondary resectability of colorectal liver metastases following neoadjuvant chemotherapy with cetuximab: The CELIM randomised phase 2 trial. Lancet Oncol 11: 38-47, 2010.

16. Van Loon K, Wigler D, Niedzwiecki D, Venook AP, Fuchs C, Blanke C, Saltz L, Goldberg RM and Meyerhardt JA: Comparison of dietary and lifestyle habits among stage III and metastatic colorectal cancer patients: Findings from CALGB 89803 and CALGB 80405. Clin Colorectal Cancer 12: 95-102, 2013.

17. Elez E, Argilés G and Tabernero J: First-line treatment of metastatic colorectal cancer: Interpreting FIRE-3, PEAK, and CALGB/SWOG 80405. Curr Treat Options Oncol 16: 52, 2015.

18. Wanebo HJ, Rao B, Pinsky CM, Hoffman RG, Stearns M, Schwartz MK and Oettgen HF: Preoperative carcinoembryonic antigen level as a prognostic indicator in colorectal cancer. N Engl J Med 299: 448-451, 1978.

19. Moertel CG, O'Fallon JR, Go VL, O'Connell MJ and Thynne GS: The preoperative carcinoembryonic antigen test in the diagnosis, staging, and prognosis of colorectal cancer. Cancer 58: 603-610, 1986.

20. Martin EW Jr, James KK, Hurtubise PE, Catalano P and Minton JP: The use of CEA as an early indicator for gastrointestinal tumor recurrence and second-look procedures. Cancer 39: 440-446, 1977.

21. Cooper MJ, Mackie CR, Skinner DB and Moossa AR: A reappraisal of the value of carcinoembryonic antigen in the management of patients with various neoplasms. Br J Surg 66: 120-123, 1979.

22. Steele G Jr, Ellenberg S, Ramming K, O'Connell M, Moertel C, Lessner H, Bruckner H, Horton J, Schein P, Zamcheck N, et al: CEA monitoring among patients in multi-institutional adjuvant G.I. therapy protocols. Ann Surg 196: 162-169, 1982.

23. Kuusela P, Jalanko H, Roberts P, Sipponen P, Mecklin JP, Pitkänen R and Mäkelä O: Comparison of CA19-9 and carcinoembryonic antigen (CEA) levels in the serum of patients with colorectal diseases. Br J Cancer 49: 135-139, 1984.

24. Filella X, Molina R, Grau JJ, Piqué JM, Garcia-Valdecasas JC, Astudillo E, Biete A, Bordas JM, Novell A, Campo E, et al: Prognostic value of CA 19.9 levels in colorectal cancer. Ann Surg 216: 55-59, 1992. 
25. Kouri M, Pyrhönen S and Kuusela P: Elevated CA19-9 as the most significant prognostic factor in advanced colorectal carcinoma. J Surg Oncol 49: 78-85, 1992.

26. Nakayama T, Watanabe M, Teramoto T and Kitajima M: CA19-9 as a predictor of recurrence in patients with colorectal cancer. J Surg Oncol 66: 238-243, 1997.

27. Prager GW, Braemswig KH, Martel A, Unseld M, Heinze G, Brodowicz T, Scheithauer W, Kornek G and Zielinski CC: Baseline carcinoembryonic antigen (CEA) serum levels predict bevacizumab-based treatment response in metastatic colorectal cancer. Cancer Sci 105: 996-1001, 2014.

28. Bramswig KH, Poettler M, Unseld M, Wrba F, Uhrin P, Zimmermann W, Zielinski CC and Prager GW: Soluble carcinoembryonic antigen activates endothelial cells and tumor angiogenesis. Cancer Res 73: 6584-6596, 2013.

29. Carmeliet P: Angiogenesis in life, disease and medicine. Nature 438: 932-936, 2005.

30. Ocvirk J, Brodowicz T, Wrba F, Ciuleanu TE, Kurteva G, Beslija S, Koza I, Pápai Z, Messinger D, Yilmaz U, et al: Cetuximab plus FOLFOX6 or FOLFIRI in metastatic colorectal cancer: CECOG trial. World J Gastroenterol 16: 3133-3143, 2010.

31. Van Cutsem E, Cervantes A, Adam R, Sobrero A, Van Krieken JH, Aderka D, Aranda Aguilar E, Bardelli A, Benson A, Bodoky G, et al: ESMO consensus guidelines for the management of patients with metastatic colorectal cancer. Ann Oncol 27: 1386-1422, 2016.
32. Cremolini C, Loupakis F, Antoniotti C,Lupi C, Sensi E, Lonardi S, Mezi S, Tomasello G, Ronzoni M,Zaniboni A, et al: FOLFOXIRI plus bevacizumab versus FOLFIRI plus bevacizumab as first-line treatment of patients with metastatic colorectal cancer: Updated overall survival and molecular subgroup analyses of the openlabel, phase 3 TRIBE study. Lancet Oncol 16: 1306-1315, 2015.

33. Sasaki Y, Osada S, Mori R, Imai H, Tanaka Y, Matsuhashi N, Okumura N, Nonaka K, Takahashi T and Yoshida K: Determining timing of hepatectomy for colorectal cancer with distant metastasis according to imaging-based tumor shrinkage ratio. Int J Med Sci 10: 1231-1241, 2013.

34. Sasaki Y, Osada S, Matsui S, Imai H, Tanahashi T, Tanaka Y, Matsuhashi N, Okumura N, Yamaguchi K and Yoshida K: Preoperative chemotherapy can change the surgical procedure for hepatectomy in patients with liver metastasis of colorectal cancer. Anticancer Res 35: 5485-5489, 2015.

35. Benson AB III, Venook AP, Cederquist L, Chan E, Chen YJ, Cooper HS, Deming D, Engstrom PF, Enzinger PC, Fichera A, et al: Colon Cancer, Version 1.2017, NCCN Clinical Practice Guidelines in Oncology. J Natl Compr Canc Netw 15: 370-398, 2017.

This work is licensed under a Creative Commons Attribution-NonCommercial-NoDerivatives 4.0 International (CC BY-NC-ND 4.0) License. 